\title{
GRAPE-SPH SIMULATIONS OF THE CHEMODYNAMICAL EVOLUTION OF DWARF GALAXIES
}

\author{
MASAO MORI ${ }^{1}$, YUZURU YOSHII ${ }^{2,3}$, TAKUJI TSUJIMOTO ${ }^{4}$ \\ AND KEN'ICHI NOMOTO ${ }^{1,3}$ \\ ${ }^{1}$ Department of Astronomy, University of Tokyo, Japan \\ ${ }^{2}$ Institute of Astronomy, University of Tokyo, Japan \\ ${ }^{3}$ Research Center for the Early Universe, \\ The University of Tokyo \\ ${ }^{4}$ National Astronomical Observatory, Japan
}

\section{Introduction}

The central concentration of the luminosity distribution in dwarf elliptical galaxies ( $\mathrm{dEs}$ ) is weaker than that of giant elliptical galaxies ( Es ). In other words, the luminosity profiles in Es follow the de Vaucouleurs' law whereas dEs have exponential luminosity profiles. Athanassoula(1994) describes the one dimensional simulations of the formation of dEs that include the feedback effects from supernovae. The model with no dark matter halo is shown to be much better agreements with the observations than with dark matter halo. However no attempt has yet been made to reproduce much lower heavy element abundances in dEs than in Es. We calculate the chemodynamical evolution of a less massive gas cloud with an $\mathrm{SPH}+\mathrm{N}-$ body three dimensional simulation code to explore the luminosity profile and chemical abundances in $\mathrm{dEs}$.

\section{Models}

The code is based on the Smoothed Particle Hydrodynamics. This code assumes that stars are formed in regions both converging and Jeans-unstable. Supernovae are treated as sources of heat, mass, and heavy elements. The gravitational forces are calculated with GRAPE-3AF.We assume that a protogalaxy is initially an isolated isothermal gas sphere, and carry out simulations including gas and stars, but no dark matter halo. 

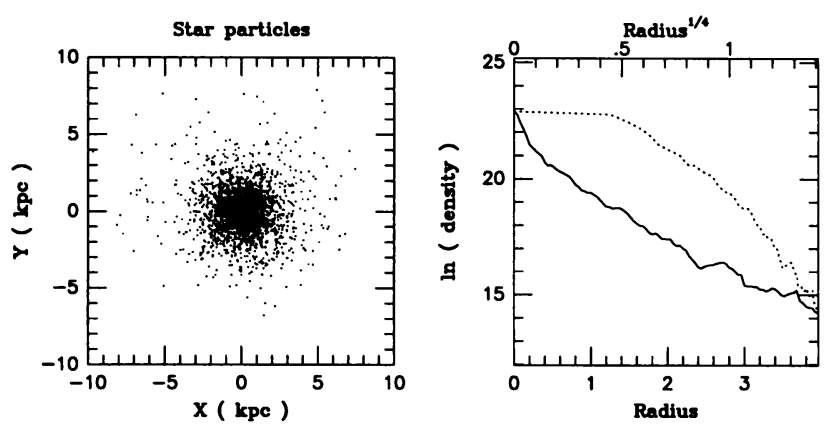

Figure 1. Left : Snapshot of stellar distribution. Right : Projected stellar density profile. The solid and the dotted lines are density profiles against the radius and the quartic root of radius, respectively.

\section{Results and Conclusions}

Our calculation indicates that the protogalaxy cloud begin to collapse due to its self-gravity. The collapse is halted by supernova driven-wind. Then the system loses a significant fraction of mass because of its small escape velocity. Figure 1 , which is a snapshot after the system reaches a quasi stationary state, shows that the projected stellar surface density profile is approximately exponential as observed in $\mathrm{dEs}$. We also find that heavy element abundance of the system is as low as $[\mathrm{Fe} / \mathrm{H}] \sim-1.5$. If the gas cloud has a smaller mass and a higher star formation rate, the stellar system would become unbound to disrupt. For a larger mass and a lower star formation rate, enough stars form before a significant mass loss from the system occurs, and a bound stellar system results eventually. Since about a half of the initial gas is blown away, the resulting stellar distribution has a weak central concentration. The chemical evolution as well as the effects of dark matter will be published elsewhere.

\section{Acknowledgements}

This work has been supported in part by the Grant-in-Aid of the Ministry of Education, Science, and Culture ( 05242102, 06233101, 07CE2002 ).

\section{References}

Deckel, A. and Silk, J. (1986) ApJ 303, 39.

Athanassoula, E. (1994) in ESO/OHP Workshop on Dwarf Galaxies., Meylan, G. \& Prugniel, P., eds., ESO, Garching, p. 525. 